\title{
CLÚSTERES Y SISTEMAS PRODUCTIVOS LOCALES \\ EN LA FRONTERA NORTE DE MÉXICO: BAJA CALIFORNIA
}

\author{
Sárah Eva Martínez Pellégrini \\ El Colegio de la Frontera Norte
}

\section{RESUMEN}

Este artículo $^{1}$ retoma el caso del estado de Baja California para discutir sus bases de desarrollo, ganador en términos de integración a América del Norte, y que hasta la fecha no parece haber logrado una reestructuración de su base económica que permita hablar de la existencia de un sistema productivo local, con cierto grado de integración. El problema fundamental de esta situación es que la economía bajacaliforniana ha mantenido un patrón de crecimiento sin tanto desarrollo hacia una competitividad basada en factores otros que su ubicación geográfica y el precio de la mano de obra. La política de desarrollo empresarial basada en clústeres, implementada en el estado a partir de 2002, podría lograr introducir algunos cambios en el sistema regional hacia la articulación de un sistema territorial con una nueva visión de competitividad, si se analiza desde el marco de las propuestas de desarrollo endógeno.

Palabras clave: clúster, política industrial, sistemas productivos locales. 


\section{ABSTRACT}

This article discusses whether Baja California, a northern border Mexican state, has an endogenous local development dynamic, or its achievements in economic growth rest on a weak economic base with a high level of exogenous dependency. Being one of the TLALCAN-winners does not seem to have endowed this region with inner articulation as to find a Local Productive System on which Baja California could ground an endogenous development model. The recent (2002) change in the regional development policy targeting «clusterization» of economic activities could contribute start a new way to talk competitivity in the region.

Key words: cluster, industrial policy, local productive systems. 


\section{INTRODUCCIÓN}

El objetivo de este artículo es presentar y analizar algunas características de las políticas de desarrollo económico en el estado fronterizo de Baja California, México, basadas en la integración de clústeres. Estos efectos se refieren específicamente al grado de consolidación de un sistema productivo regional con cierto nivel de organización interna particular y un modelo de desarrollo regional concreto, fundamentados en la empresarialidad local y capaces de recuperarla y mejorarla.

Baja California es de los estados «ganadores» del proceso de integración de México con Estados Unidos, ${ }^{2}$ situación que deriva en gran parte de una previa integración de facto con Estados Unidos a través de las maquiladoras, ensambladoras, localizadas en el estado. Estas empresas explican gran parte del empleo industrial y las exportaciones de la región. El impulso industrial descrito ha generado una dinámica de crecimiento económico inducido y alimentado por factores exógenos, sin que se hayan detonado dinámicas locales espontáneas que permitan hablar de un proceso de desarrollo endógeno o local. La reciente política de fomento del desarrollo de clústeres del gobierno del estado podría ser un elemento que modifique esta situación en los próximos años, aunque aún es pronto para poder hablar de resultados consolidados.

En el texto se discute si los sectores en que está especializada la región, aquellos que explican su dinamismo económico, presentan una estructura integrada sectorial y territorialmente que se pueda considerar como un sistema productivo local articulado con cierto grado de endogenidad. Es decir, si a partir de su situación geográfica privilegiada y el fomento de los clústeres en el estado, Baja California presenta una senda de 
movilización de su recurso empresarial tendente a la consolidación de un sistema regional con mayor equilibrio de componentes exógenos y endógenos.

La hipótesis de partida del planteamiento es que una región ganadora en un proceso de integración de las características del Tratado de Libre Comercio de América del Norte, TLCAN, Baja California, no es una región que está consolidando un modelo de desarrollo que articule su base productiva con un esquema de organización territorializado. En otras palabras, el sistema productivo carece de una organización orientada a la capacidad de respuesta a los cambios del mercado y las circunstancias económicas, a la capitalización de la capacidad emprendedora local y a la generación de ventajas competitivas específicas, que le permitan determinar su propia senda de desarrollo. En este sentido, no se puede hablar aún de la existencia de un proceso de reestructuración y reorganización en el ámbito regional acorde con los planteamientos de las teorías del desarrollo endógeno.

Discutir esta hipótesis equivale a refrendar que la tasa de crecimiento es información insuficiente para determinar que una economía regional, o nacional, tiene características estructurales «sanas» que garanticen su competitividad y por tanto su capacidad de crecer en el tiempo. La aproximación a través del análisis de factores asociados con el desarrollo, como son la organización de la base económica y su articulación con otros actores regionales, resultan indicadores más adecuados para conocer la situación de una economía frente al entorno y al futuro. Esta conclusión también relativiza la utilidad de los estudios de convergencia como instrumentos únicos de evaluación de la situación y logros en materia de desarrollo económico. 
El texto inicia con una presentación del marco teórico y los conceptos utilizados para el análisis, sigue la presentación del caso de Baja California y concluye con algunas reflexiones de considerado interés.

\section{LAS TEORÍAS Y CONCEPTOS MARCO DE LA DISCUSIÓN}

El eje del análisis que se propone es la organización espacial del sistema económico, y a partir de ahí la integración del sistema productivo local y su inclusión en el sistema regional. Partimos de que la economía regional puede concebirse como un sistema de desarrollo, cuya estructura productiva queda definida por la diversidad de sus componentes y su grado de integración (Perrin 1974); la estructura productiva regional es un conjunto específico de actividades productivas localizadas en un espacio regional, cuya dinámica impone sus propias limitaciones a la organización espacio-económica del sistema general y tiene ciertos impactos directos en la organización social.

No se puede avanzar en la definición de las dinámicas y características del territorio únicamente a partir de los elementos endógenos mencionados en el planteamiento anterior, además es necesario recuperar de forma explícita las principales líneas de interacción de ese sistema regional con «el exterior», puesto que en algunos casos estas pueden tener incluso mayor incidencia en la organización interna que los factores endógenos. En este sentido es importante recuperar las aportaciones sobre los impactos territoriales de la integración económica, sobre todo para el análisis de las regiones fronterizas. De hecho las evidencias de interacción e influencia mutua entre regiones nacionales y supranacionales conduce directamente a la discusión de la paradoja territorial mencionada por Precedo (2004), en la que se recuperan espacios de acción 
locales y regionales, precisamente, como forma más efectiva de respuesta a los efectos del funcionamiento del «gran sistema» mundial.

Una aportación particularmente relevante para retomar esta paradoja territorial es el cambio del viejo al nuevo regionalismo que se plantea en el ámbito de las relaciones internacionales. En la medida en que los procesos de integración y globalización se acentúan, sobre todo los primeros, las diferencias entre los territorios van quedando circunscritas en mayor medida a sus características endógenas, y se desdibujan con el tiempo las derivadas de lo que podríamos denominar el efecto nación.

El nuevo regionalismo implica subrayar el análisis de la integración económica como regional, por lo tanto se habla de la constitución de grandes zonas integradas por procesos de asociación que resultan en entornos suficientemente homogéneos funcionalmente. ${ }^{3}$ Esta visión compleja, que involucra varias dimensiones de interrelación, es la que permite afirmar el acercamiento de las esferas regionales, supranacionales y subnacionales, en ciertos aspectos característicos que les dan carácter de complementariedad. Su similitud deriva de la generalización de la lógica de competencia y desarrollo de ventajas competitivas en los diferentes niveles de definición de espacios, o regiones, sean subnacionales, nacionales o supranacionales.

Lo que se retoma de la discusión del impacto de la integración económica sobre el bienestar son los efectos dinámicos de tales procesos de integración, ya que estos nos parecen los más relevantes en el contexto de la mejora de los sistemas regionales o locales, mediante mecanismos de ganancias o pérdidas sistémicas de competitividad y por tanto beneficios a medio y largo plazo. Tienen que ver con los ajustes estructurales que se producen en las economías tras ser sometidas a mayores grados de competencia, 
que pueden modificar las trayectorias de crecimiento y desarrollo económicos y sobre todo sus formas de organización y capacidades de respuesta.

Si hasta ahora hemos hablado de los factores exógenos más asociados con las visiones tradicionales del desarrollo regional y los procesos de integración y globalización, es necesario dar el siguiente paso y profundizar en cómo se incorporan al análisis las especificidades territoriales. La forma de incidencia de los factores exógenos depende de las características propias de la región y en particular de su potencial endógeno. Ese potencial incluye como factores de fortaleza o debilidad local una multitud de elementos que abarca desde los tangibles hasta los intangibles de los recursos y la organización del sistema local. Colletis y Pecqueur (1993) los agrupan en cinco que podríamos considerar endógenos: 1. el soporte territorial como proveedor de recursos, 2. el modelo de especialización productiva local, 3. la localización y articulación de las industrias, 4. las características laborales y emprendedoras de la población, y 5. la existencia de centros y organizaciones capaces de asumir el liderazgo; se añade uno de interacción con otros sistemas: 6. la integración a otros mercados. ${ }^{4}$ Estos seis puntos resumen a grandes rasgos las líneas fundamentales de acercamiento al diagnóstico de las características propias de un sistema regional; y entre los de carácter endógeno, el modelo de especialización productiva local, la localización y articulación de las industrias, y las características laborales y emprendedoras de la población, son los rasgos que definen la iniciativa empresarial regional, elemento central de la discusión planteada en este texto.

Debe considerarse que así como estas características pueden encerrar un potencial también pueden ser limitantes para el desarrollo de un territorio, y en ese sentido habrá que complementar lo anterior considerando la factibilidad de cambio o reestructuración 
productiva $^{5}$ factible para cada caso concreto. Esta dependerá de 1) la estructura económica y organización de la producción existentes, 2) la evolución del sistema tecnológico, 3) el mercado laboral y las relaciones industriales, 4) los actores y estructuras sociales, 5) las características del mercado y las formas de competencia, 6) la fluidez de circulación de la información, y 7) las instituciones reguladoras existentes.

Los distritos industriales son un antecedente importante de nuestro planteamiento, su técnica productiva depende de variables del entorno y no exclusivamente del precio de los factores productivos. Garofoli (1992) destaca como elementos básicos de estos sistemas la existencia de una cultura del trabajo entre los diversos establecimientos que los integran, una fuerte especialización productiva, la intervención de una pluralidad de agentes locales, el establecimiento de un sistema eficiente de trasmisión de la información a escala local, el alto grado de calificación de la mano de obra, y la proliferación de relaciones personales entre los agentes económicos. De nuevo varios de estos puntos se encuentran relacionados de manera directa con las características del empresariado y las empresas locales.

La principal aportación de los enfoques de desarrollo local es buscar planteamientos integrales y territoriales de desarrollo cuyos objetivos vendrán definidos por las especificidades de cada región. Más allá del marco teórico del que se parte, la evaluación de las iniciativas debe hacerse en función de los objetivos que explícitamente plantean y del contexto al que se aplican. En el caso de América Latina, por ejemplo, entre los principales objetivos de las iniciativas de desarrollo económico local aparecen los siguientes (Alburquerque, en Aghon et al. 2001: 292-293): 
- Diversificación productiva basada en los recursos endógenos, locales.

- Articulación público-privada para promover la innovación productiva y empresarial.

- Cooperación entre municipios para lograr mejores resultados en desarrollo.

- Estímulo de semilleros de empleo locales y nuevas fuentes de ingreso.

- Apoyo financiero a las micro, pequeñas y medianas empresas.

- Promoción de la competitividad sistémica territorial.

- Iniciativas relacionadas con la sustentabilidad.

A pesar de su generalidad podemos identificar claramente que estos objetivos abarcan los diferentes aspectos considerados centrales para endogenizar el desarrollo. Merecen particular atención los puntos de diversificación productiva, promoción de la competitividad sistémica territorial e iniciativas para la sustentabilidad, a parte de los riesgos atribuidos a las iniciativas orientadas al fomento de agrupamientos industriales. ${ }^{6}$

\section{LA REGIÓN BAJACALIFORNIANA Y SU ESTRATEGIA DE DESARROLLO}

\section{PoLÍTICAS NACIONALES DE DESARROLLO ECONÓMICO Y SU IMPACTO REGIONAL EN MÉXICO}

Ni la política de sustitución de importaciones ni la de apertura al exterior aplicadas en México desde los años cincuenta hasta la fecha han contribuido a un equilibrio social o territorial. Estos resultados eran previsibles a partir de las premisas y orientación de ambas hacia la consecución de mayores niveles de crecimiento de los que se supone derivarían naturalmente la redistribución y el desarrollo, sin ningún otro tipo de medida equilibradora. Pero sobre todo dado que de forma natural las políticas diseñadas de forma muy vertical 
reforzaron los patrones existentes provocando una situación como la descrita por la teoría de la causación acumulativa. En el país, el desarrollo y el crecimiento se concentraron en las grandes ciudades y sus áreas de influencia directa, posteriormente y como resultado de la aparición de las deseconomías de aglomeración y del avance en las comunicaciones y trasportes, se incorporaron a la dinámica de crecimiento algunas áreas aledañas a las anteriores.

Las características de México, por su extensión y heterogeneidad, de partida dificultan en gran medida siquiera la homogeneización de condiciones mínimas. Algunos de los factores que lo explican son las características demográficas, climatológicas, culturales y de desarrollo previas. Las diferencias entre las regiones de México superan en algunos aspectos a las divergencias entre los países de la Unión Europea —al menos hasta la última ampliación.

La conclusión que se saca de las características de la única política que se podría considerar más regional o territorial, la urbana, es que no tenía tintes de política de desarrollo sino de provisión de servicios a la población, y en ese contexto en algún momento llegó a plantear una revisión de las dimensiones urbanas y de los problemas de gran crecimiento de pocos núcleos de población, sin plantear una estrategia real de desarrollo o reforma del patrón de asentamiento de población ni de la dinámica productiva imperantes. Derivado de lo anterior, no se puede hablar hasta fechas muy recientes —alrededor del año 2000 — de una visión de desarrollo regional, sino de crecimiento económico nacional, por un lado, y de solución de problemas urbanos, por otro. Esta situación se refleja claramente en el mapa de actores relevantes en el desarrollo en México que han sido tradicionalmente el gobierno central y los municipios grandes; los 
gobernadores o gobiernos estatales aparecen en escena cuando surgen las peticiones de redistribución de las participaciones federales en la segunda mitad de los años ochenta. Esta situación es particularmente preocupante en un contexto en el que aún no se ha dado la transición de las estructuras centrales hacia un papel de árbitro y nivelador de las diferencias de desarrollo entre regiones, en lugar de ser interventor directo en las iniciativas de desarrollo.

El país presenta una situación general de estancamiento de la convergencia, que desde nuestro punto de vista y según los datos ${ }^{7}$ es más bien de polarización de la situación de desequilibrio entre las «locomotoras» y los «furgones de cola» en lo que a desarrollo se refiere. ¿Qué agrava esta situación en el caso de países de desarrollo tardío como México? Que en ellos los indicadores de condiciones de vida por grupos están desplazados hacia los extremos inferiores, y por lo tanto en estos casos los perdedores o casos extremos se ubican por debajo de las líneas aceptables de nivel de vida, en situaciones que amenazan sus posibilidades de sobrevivir.

Retomando las disparidades regionales por entidades federativas, las gráficas 1 y 2 presentan los resultados de un análisis de $\beta$ condicionada para los estados mexicanos en el periodo 1980-1998 (Martínez 2006), que se subdivide en dos etapas correspondientes a las políticas de cierre del país y de apertura internacional, respectivamente. Para el primer periodo, que abarca la mayor parte del modelo de industrialización por sustitución de importaciones, ISI, y una pequeña parte del periodo de crisis económica, existe una clara convergencia $\beta$ absoluta, ya que los estados con menor ingreso per cápita en 1975 : Tlaxcala, Oaxaca, Zacatecas, San Luis Potosí, Nayarit y Durango, fueron los que presentaron mayores tasas de crecimiento. 
Gráfica 1.

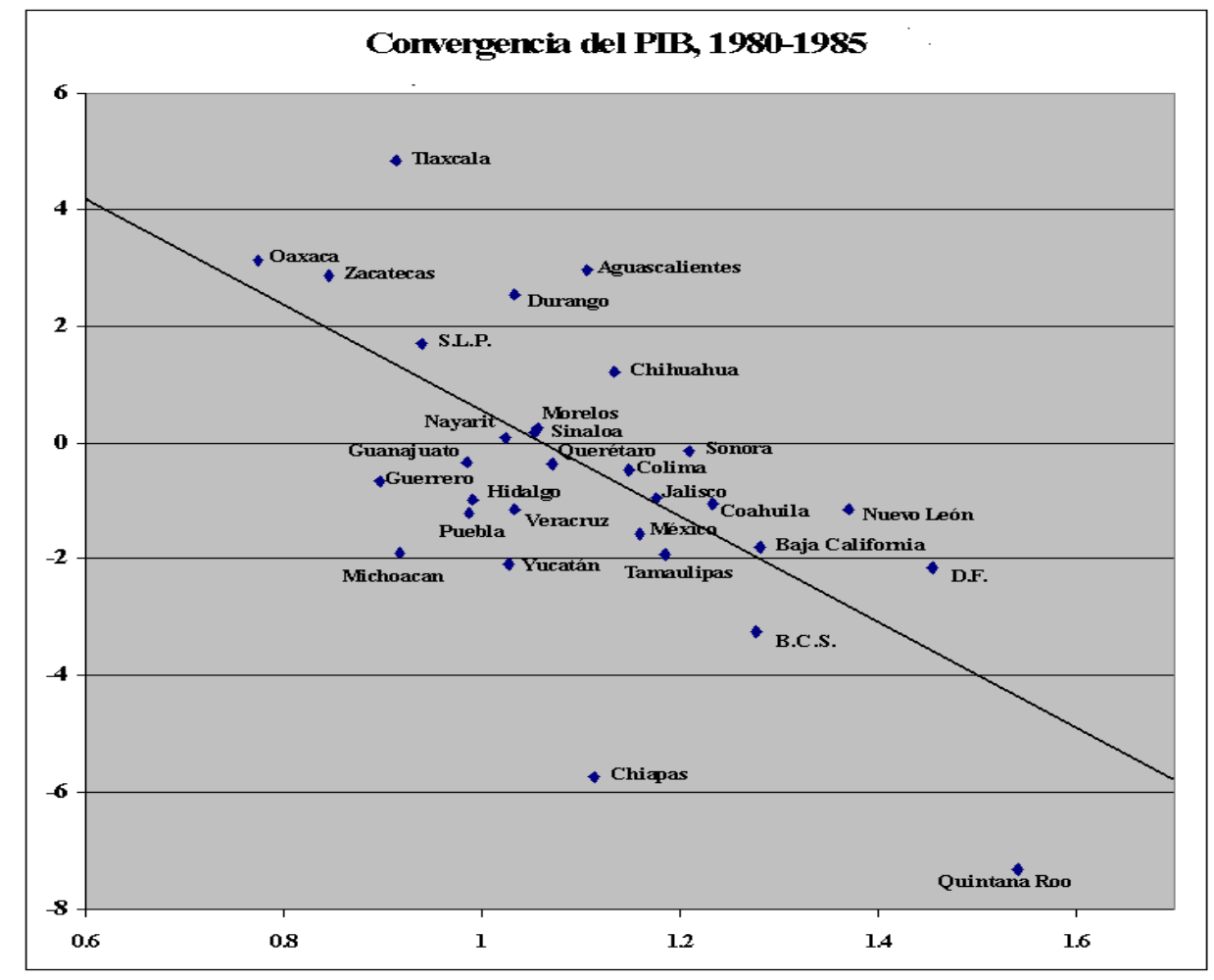

Fuente: Sárah Eva Martínez Pellégrini 2006.

La Gráfica 2 evidencia, con datos del producto interior bruto para los estados mexicanos en el periodo 1985-1998, una clara divergencia $\beta$ absoluta. Las entidades federativas con menor ingreso per cápita en 1985: Tlaxcala, Oaxaca, Zacatecas, Nayarit y Durango, crecen más despacio que el promedio nacional, mientras que las regiones con mayor ingreso per cápita en 1980: Baja California, Tamaulipas, Nuevo León y D. F., lo hacen a tasas superiores. 


\section{Gráfica 2.}

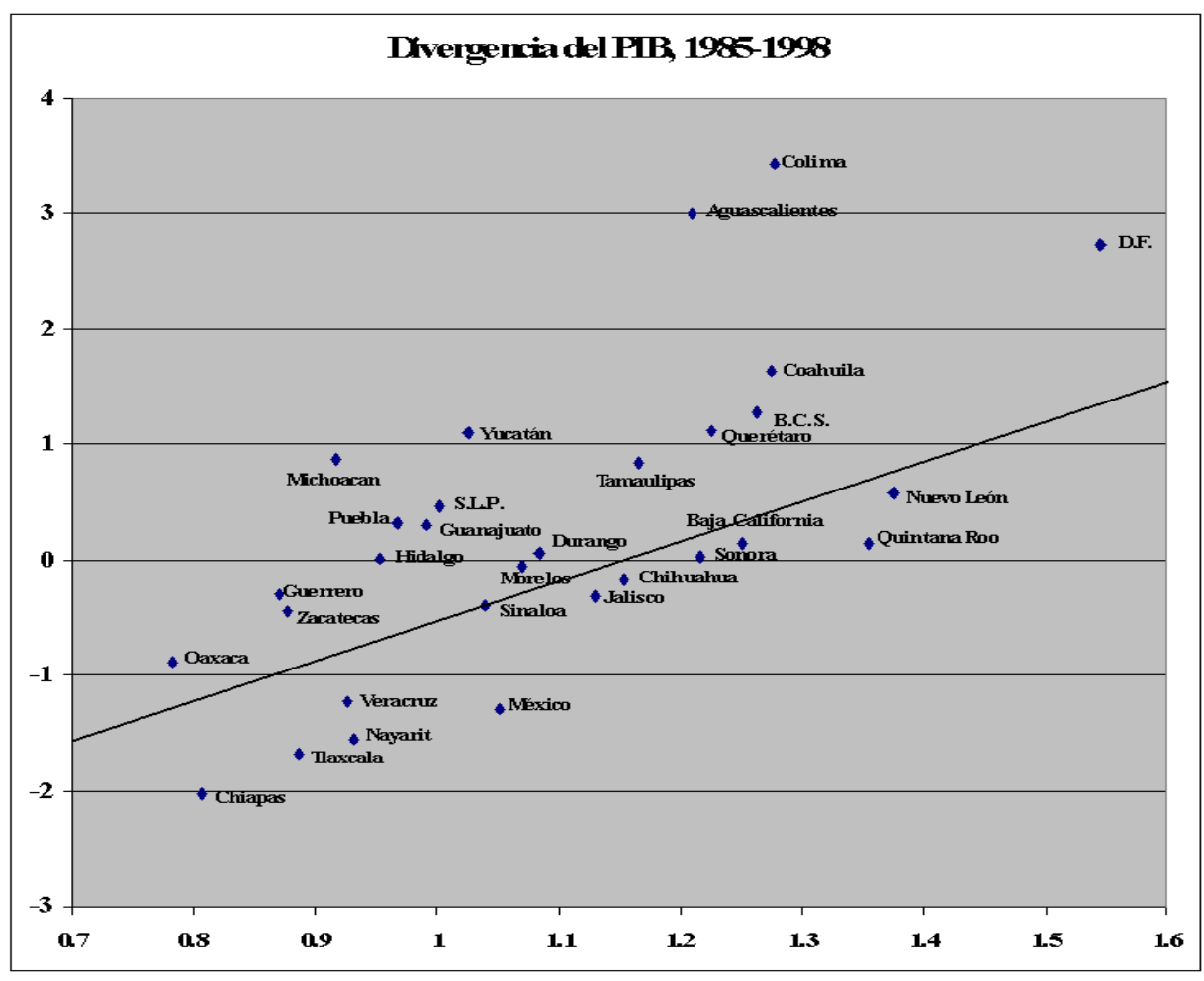

Fuente: Sárah Eva Martínez Pellégrini 2006.

La conclusión es que se constata la existencia de dos etapas claramente diferenciadas respecto a la convergencia en los niveles de ingreso per cápita en el periodo 1980-1998; una primera, 1980-85, con acercamiento de la mayoría de los estados más rezagados al promedio nacional; y posteriormente otra, 1985-1998, de progresiva divergencia después de la apertura comercial iniciada en 1985. Con estos resultados se puede concluir que la política de Industrialización Orientada hacia el Exterior, IOE, antecedente del TLCAN, ha tenido efectos negativos en cuanto a la convergencia regional entre los estados mexicanos. Sin embargo, es necesario matizar esta afirmación profundizando algo más en el proceso mediante la estimación de la convergencia condicional. 
El otro gran problema es que las disparidades entre regiones y entre grupos sociales en estos países en desarrollo, caracterizados por sus menores niveles de estructuración social, es mucho mayor que en países más desarrollados. ${ }^{8}$

\section{LOS CLÚSTERES COMO BASE DEL SISTEMA PRODUCTIVO LOCAL EN BAJA CALIFORNIA}

Centrándonos ahora en la dinámica económica del estado de Baja California la conclusión general a la que se llega es que esta región «ganadora» tras el proceso de apertura es una zona de crecimiento industrial con subvenciones exteriores, ${ }^{9}$ como lo demuestran su potencial de desarrollo, el tipo de reestructuración requerida por los problemas locales, el modelo de desarrollo en que se basa la economía local, y la experiencia adquirida en el manejo de los instrumentos de desarrollo.

Aunque la participación estatal en el producto interno bruto nacional ha tenido ligeros aumentos en la última década (Cuadro 1), si retomamos los índices de volumen físico de la producción manufacturera el estado ha tenido un crecimiento de la actividad muy superior al promedio nacional. Este dato es un indicador de que Baja California presenta un retroceso en el valor de su producción y por tanto se sitúa en los eslabones más bajos de la cadena de producción. Este resultado contradice la visión de que la maquiladora esté contribuyendo a la reestructuración del sistema industrial local hacia una mayor competitividad tecnológica y mejora del capital humano regional. 
Cuadro 1. Participación en el PIB nacional por municipios

\begin{tabular}{|c|c|c|c|c|c|c|c|c|c|c|}
\hline$A \tilde{n} o s$ & \multicolumn{10}{|c|}{ Municipios } \\
\hline & \multicolumn{2}{|c|}{ Tijuana } & \multicolumn{2}{|c|}{ Mexicali } & \multicolumn{2}{|c|}{ Ensenada } & \multicolumn{2}{|c|}{ Tecate } & \multicolumn{2}{|c|}{ Baja California } \\
\hline & & $\%$ & & $\%$ & & $\%$ & & $\%$ & & $\%$ \\
\hline 1994 & $19,568,862$ & 1.50 & $12,563,666$ & 0.96 & $1,720,893$ & 0.13 & $1,218,294$ & 0.09 & $38,071,715$ & 2.91 \\
\hline 1995 & $27,478,856$ & 1.64 & $17,642,067$ & 1.05 & $6,629,140$ & 0.39 & $1,710,746$ & 0.10 & $53,460,809$ & 3.18 \\
\hline 1996 & $38,097,563$ & 1.66 & $23,221,372$ & 1.01 & $8,708,014$ & 0.38 & $2,539,837$ & 0.11 & $72,566,786$ & 3.16 \\
\hline 1997 & $50,153,250$ & 1.58 & $30,569,600$ & 0.96 & $11,463,600$ & 0.36 & $3,343,550$ & 0.11 & $97,637,698$ & 3.39 \\
\hline 1998 & $61,620,825$ & 1.60 & $37,559,360$ & 0.98 & $14,084,760$ & 0.37 & $4,108,055$ & 0.11 & $119,420,226$ & 3.39 \\
\hline 1999 & $75,557,175$ & 1.64 & $46,050,240$ & 1.00 & $17,268,840$ & 0.38 & $5,036,745$ & 0.11 & $148,810,050$ & 3.53 \\
\hline 2000 & ND & & ND & & ND & & ND & & $180,879,629$ & 3.63 \\
\hline 2001 & ND & & ND & & ND & & ND & & $183,592,039$ & 3.48 \\
\hline 2002 & ND & & ND & & ND & & ND & & $189,341,710$ & 3.30 \\
\hline 2003 & ND & & ND & & ND & & ND & & $210,633,123$ & 3.37 \\
\hline 2004 & ND & & ND & & ND & & ND & & $244,088,677$ & 3.50 \\
\hline 2005 & ND & & $\mathrm{ND}$ & & ND & & ND & & $264,439,299$ & 3.54 \\
\hline 2006 & ND & & ND & & ND & & ND & & $294,838,022$ & 3.60 \\
\hline
\end{tabular}

Fuente: INEGI.

El potencial de desarrollo económico de la región está por lo tanto fuertemente anclado en el reciente desarrollo industrial que se fomentó a partir de la instalación de maquiladoras en el estado durante los años 60 del siglo pasado como resultado del régimen de zona libre. En este sentido la vocación en el estado es claramente industrial en términos de los activos o factores productivos que se están explotando de hecho. Los sectores manufactureros con mayor peso desde principios de los años 90 son «productos metálicos, maquinaria y equipo» con participaciones crecientes de entre $40 \%$ y $51 \%$ de la producción manufacturera; «alimentos, bebidas y tabaco» ha ido de 21 a $14.5 \%$ de la participación; 
«minería no metálicos excepto derivados del petróleo» y «madera y sus productos» pasaron de representar alrededor de 7\% de la producción estatal a 5\%, y «químicos, derivados del petróleo, caucho y plásticos» ascendió de $4.8 \%$ a 5.57 ; por último, «textiles, vestido y cuero» partieron de 3.33 y llegaron a 4.63\%. La evolución sectorial del estado en el periodo 1993-1999 permite establecer una clara distinción entre actividades predominantemente organizadas, como maquiladoras y conexas que han crecido en el periodo, y aquellas dirigidas al abasto local o nacional que han decrecido.

Los servicios complementan la actividad manufacturera como recurso o factor por explotar, y aunque aún se centran en el comercio han ido evolucionando hacia servicios turísticos asociados con algunos servicios profesionales de alto valor añadido como los médicos.

En general los últimos años han dado indicios de cierta reestructuración de la base productiva regional con al afianzamiento de los sectores donde hay maquiladoras, la contracción de los sectores tradicionales locales y el surgimiento, aún a pequeña escala, de nuevos sectores competitivos y dirigidos a mercados muy concretos. La maquiladora presentó durante todo el periodo 1986-2000 tasas de crecimiento del empleo y del número de plantas superiores al promedio nacional, además mantuvo su generación de divisas a tasas de crecimiento superiores a $6 \%$ anual. Por lo tanto mantuvo buenos resultados en sus dos contribuciones básicas a la economía: empleo y divisas; sin embargo, no encontramos evidencia de que esas maquiladoras estén teniendo otro tipo de derramas estructurales u organizativas en el sistema local.

Este proceso podría significar una polarización del sistema económico regional entre sectores de ventaja comparativa asociados con la maquiladora que absorben la mayor 
parte de los empleos y mantienen bajos niveles de valor añadido, y sectores minoritarios en empleo de alto valor añadido que permiten el desarrollo de áreas competitivas en el estado.

Cuadro 2. Industria maquiladora de exportación por municipio, B. C.

\begin{tabular}{|c|c|c|c|}
\hline & Mexicali \% & Tecate \% & Tijuana \% \\
\hline \multicolumn{4}{|c|}{ Número de establecimientos } \\
\hline 1995 & 16.60 & 11.11 & 65.43 \\
\hline 1996 & 16.12 & 10.71 & 66.62 \\
\hline 1997 & 16.37 & 10.62 & 66.26 \\
\hline 1998 & 16.80 & 11.00 & 65.52 \\
\hline 1999 & 16.28 & 11.12 & 65.21 \\
\hline 2000 & 15.93 & 11.33 & 64.70 \\
\hline 2001 & 15.38 & 11.36 & 64.94 \\
\hline 2002 & 14.85 & 12.09 & 63.94 \\
\hline 2003 & 14.75 & 12.27 & 63.96 \\
\hline 2004 & 14.51 & 12.36 & 64.74 \\
\hline 2005 & 14.95 & 12.74 & 63.57 \\
\hline 2006 & 14.79 & 12.80 & 63.69 \\
\hline \multicolumn{4}{|c|}{ Personal ocupado } \\
\hline 1995 & 19.04 & 6.29 & 70.55 \\
\hline 1996 & 20.60 & 5.24 & 70.09 \\
\hline 1997 & 21.43 & 4.50 & 69.31 \\
\hline 1998 & 22.03 & 4.70 & 68.29 \\
\hline 1999 & 21.97 & 4.90 & 67.67 \\
\hline 2000 & 22.62 & 4.37 & 67.59 \\
\hline 2001 & 21.94 & 4.21 & 68.11 \\
\hline 2002 & 22.89 & 4.11 & 66.71 \\
\hline 2003 & 23.68 & 4.15 & 65.84 \\
\hline 2004 & 22.88 & 4.17 & 67.22 \\
\hline 2005 & 22.59 & 4.18 & 67.52 \\
\hline 2006 & 21.79 & 4.32 & 68.51 \\
\hline \multicolumn{4}{|c|}{ Valor agregado (3) } \\
\hline 1995 & 19.04 & 5.59 & 72.33 \\
\hline 1996 & 20.70 & 4.63 & 71.82 \\
\hline 1997 & 22.72 & 3.94 & 69.81 \\
\hline 1998 & 22.20 & 3.64 & 70.50 \\
\hline 1999 & 22.15 & 3.76 & 69.97 \\
\hline 2000 & 23.40 & 3.30 & 69.57 \\
\hline 2001 & 24.96 & 3.50 & 67.55 \\
\hline 2002 & 26.29 & 3.49 & 65.89 \\
\hline 2003 & 30.66 & 3.52 & 61.57 \\
\hline 2004 & 29.45 & 3.59 & 63.08 \\
\hline 2005 & 28.63 & 3.82 & 63.46 \\
\hline 2006 & 26.36 & 4.11 & 65.81 \\
\hline
\end{tabular}

Fuente: Estadísticas económicas INEGI, Industria Maquiladora de Exportación, varios años . 
Esta polarización entre tipos de unidades productivas se repite en la distribución espacial de actividad, ya que el estado de Baja California presenta una clara distinción entre los dos municipios más poblados y dinámicos: Tijuana, que se puede considerar el centro económico; y Mexicali, que es la capital del estado. Hay otros tres municipios: Ensenada, Rosarito y Tecate, de menores dimensiones poblacionales y menor tasa de crecimiento (ver Cuadro 2). Tijuana aporta alrededor de 50\% del PIB estatal, y Mexicali aproximadamente $30 \%$.

Los problemas económicos fundamentales de la región, derivados del modelo de desarrollo en que se basa la economía local, requieren la consolidación del sistema regional para lograr cierto nivel de control endógeno de su crecimiento y desarrollo. Hasta hace apenas unos años la única política de desarrollo regional existente era la promoción de inversión extranjera sin discriminación de sectores o actividades, es decir, sin una visión de construcción de un sistema productivo con cierta orientación para un desarrollo a largo plazo. El modelo de desarrollo estaba totalmente dirigido hacia la atracción de grandes empresas extranjeras por la disponibilidad de mano de obra barata y la proximidad al mercado estadounidense, además de alguna otra concesión, fundamentalmente fiscal, negociada para cada caso concreto. Todo ello explica el crecimiento sostenido de los sectores con presencia maquiladora y la contracción o estancamiento de los demás sectores productivos. El origen del capital invertido en la zona es mayoritariamente estadounidense, seguido del japonés y el coreano.

Tijuana concentra la mayor parte de las maquiladoras, alrededor de $60 \%$ del total estatal, y aunque los demás municipios mantienen en general sus tasas de participación en Ensenada, municipio en el que había otro tipo de industrias en crisis, está aumentando la 
proporción de maquiladoras. Es decir, el modelo de utilizar la maquiladora como generadora de empleo e industria sigue siendo predominante, lo cual es en muchos casos una medida cortoplacista para paliar situaciones de estancamiento económico como la ensenadense.

El manejo local-regional de los instrumentos de desarrollo ha sido bastante reducido, salvo en el caso de las acciones orientadas a la captación de inversión extranjera ya citadas.

Desde aquí se detecta la escasa articulación del sistema local, ya que la política de desarrollo regional se puede considerar muy limitada por no decir inexistente. La atracción de inversión estaba mediada por las iniciativas nacionales de apoyo a la industria maquiladora en general y a algunos sectores considerados importantes, también en el ámbito nacional, sin que se incorporasen las vocaciones o los potenciales regionales específicamente. Este primer aspecto de desarticulación implica dos niveles: 1. la desconexión de las autoridades locales respecto al proyecto económico y de desarrollo de la región, y 2. la desarticulación de la base productiva que se crea en la región incapaz de integrar a los actores entre sí, particularmente los exógenos y endógenos.

Otro de los determinantes de la organización productiva regional será los rasgos propios de su sistema productivo. Partiendo de una aproximación a las economías internas y externas, a partir del trabajo de campo realizado, ${ }^{10}$ encontramos que el sistema genera escasas economías externas y que en la mayoría de los casos las plantas están aisladas, pero que en el caso de las grandes empresas extranjeras, estas tienen economías internas elevadas y tienden a una fuerte integración vertical de funciones. Podemos afirmar que existen dos dinámicas dominantes, una de PYMES locales desarticuladas y orientadas al 
mercado regional con algunas excepciones de empresas que exportan o proveen a las empresas mayores, y la otra que es la de las grandes empresas extranjeras que funcionan prácticamente como enclaves y cuyos insumos locales son el empleo y $3 \%$ de los otros recursos, que los constituyen mayoritariamente consumibles para la operación de la planta. Estos últimos no son precisamente los que podrían detonar procesos de complementariedad o de integración de cadenas productivas.

Si retomamos la propuesta (Vázquez 2002) de análisis del sistema productivo local, SPL, a partir de dos ejes: el nivel de integración territorial de las empresas y la organización reticular o en redes, que se resume en el Esquema 1; las empresas del estado tienden a situarse en el modelo de enclaves o de empresas externas integradas débilmente en la cadena de valor local. En este sentido el esfuerzo de «clusterización» se presenta como una alternativa para, por un lado, integrar a los productores externos al sistema local y por tanto enraizarlos o territorializarlos, y por otro para generar un modelo más cercano a la organización reticular de los actores productivos, ya sean locales o foráneos, en lugar del actual esquema jerárquico. Estaríamos ante una situación de apoyo a clústeres naturales en formación y de generación de otros inducidos o forzados según la terminología de PachecoVega (2007).

Esquema 1. Organización y territorialización de las empresas

\begin{tabular}{|c|c|c|c|}
\hline \multirow{4}{*}{$\begin{array}{l}\text { No integración } \\
\text { territorial de } \\
\text { las empresas }\end{array}$} & \multicolumn{2}{|c|}{ Redes de empresas } & \multirow{4}{*}{$\begin{array}{l}\text { Integración } \\
\text { territorial de las } \\
\text { empresas }\end{array}$} \\
\hline & $\begin{array}{c}\text { Sistemas locales integrados en } \\
\text { las hileras de producción de } \\
\text { otras cadenas }\end{array}$ & $\begin{array}{l}\text { Sistemas locales integrados en } \\
\text { las cadenas de valor locales }\end{array}$ & \\
\hline & Enclaves & $\begin{array}{l}\text { Empresas externas integradas } \\
\text { en la cadena local de valor }\end{array}$ & \\
\hline & \multicolumn{2}{|c|}{ Modelo jerárquico de organización } & \\
\hline
\end{tabular}

Fuente: Vázquez 2002. 
Respecto al empleo en general existen niveles de rotación laboral significativos de entre 20 y $30 \%$ de los empleados anualmente. ${ }^{11}$ Aunque todos los sectores presentan situaciones similares, para plásticos y autopartes esta situación resulta problemática, lo cual es explicable por el hecho de que en estos sectores los trabajadores son formados en la propia empresa en actividades especializadas.

Los principales problemas detectados para el mercado laboral siguen relacionados con aspectos como la rotación, el absentismo laboral y la falta de trabajadores cualificados. Por lo tanto todavía no se ha consolidado la calidad del recurso humano como una de las externalidades de la localización cercana de industrias. El único caso en que parece existir esa reserva de trabajo es para el sector eléctrico-electrónico. Estos resultados pueden relacionarse con la dinámica poblacional del estado en general y de Tijuana en particular, donde el crecimiento demográfico se explica por la llegada de migrantes en busca de empleo, ya sea en la frontera o en Estados Unidos.

Retomando las relaciones entre empresas y el grado de aglomeración espacial la industria bajacaliforniana es dispersa y más jerárquica que cooperativa. Las redes son débiles e incipientes, y lo que se puede encontrar es una aglomeración de empresas concentradas en el territorio sin gran interrelación entre ellas. Existen pocas relaciones de interdependencia y por tanto no podemos hablar de que haya redes de actores múltiples que interactúan y reaccionan a las acciones de los demás miembros de la red. Las redes más articuladas son las que responden a alguna relación jerárquica entre las empresas por diferentes motivos de control de recursos.

A pesar de que los sectores que se seleccionaron son aquellos en los que hay cierto grado de especialización regional, que tienen coeficientes de localización 
superiores a 1 (ver Cuadro 3), y con interrelaciones con otras actividades - vinculados según la matriz input/output-, no se confirma la existencia de interrelaciones productivas de subcontratación o cooperación ni la existencia de cooperación informal significativa. No hay complementariedad entre las industrias, aunque el sector textil y el automotriz presentan ciertos mecanismos de colaboración interempresarial.

Cuadro 3. Sectores de actividad con coeficientes de localización mayores a 1 en Baja California

\begin{tabular}{|c|c|c|c|c|}
\hline & Sectores de actividad & $\begin{array}{c}\text { Coeficiente de } \\
\text { localización }\end{array}$ & $\begin{array}{c}\text { Número de } \\
\text { establecimientos }\end{array}$ & $\begin{array}{c}\text { Volumen de } \\
\text { empleo }\end{array}$ \\
\hline & Total para la industria manufacturera & & 4,813 & 248,458 \\
\hline 21 & Cerveza & 1.25 & $*$ & 407 \\
\hline 13 & Industria del vestido y textiles & 1.09 & 119 & 3,411 \\
\hline 37 & Resinas sintéticas y fibras artificiales & 0.87 & 18 & 562 \\
\hline 48 & Muebles y accesorios metálicos & 1.23 & 87 & 3,218 \\
\hline 57 & Carrocerías e industria de autopartes & 1.79 & 91 & 12,569 \\
\hline 45 & Otros prod. de minerales no metálicos & 1.57 & 366 & 5,829 \\
\hline 46 & Industrias básicas del hierro y acero & 1.01 & 67 & 1,509 \\
\hline 40 & Otros productos metálicos & 1.27 & 145 & 13,389 \\
\hline 64 & Trasporte & 1.74 & 9 & 4,208 \\
\hline 54 & Equipos y accesorios electrónicos & 1.93 & 147 & 57,289 \\
\hline 43 & Vidrio y sus productos & 1.22 & 45 & 1,818 \\
\hline 42 & Artículos de plástico & 1.02 & 130 & 17,142 \\
\hline 50 & Maquinaria y equipos electrónicos & 1.28 & 102 & 18,690 \\
\hline
\end{tabular}

* Información no disponible por cuestiones de secreto estadístico.

Fuente: tomado de Martínez 2006. Elaborado con información de Censos Económicos, 1998.

La mayoría de los insumos de producción son importados, tanto materias primas como componentes y maquinaria. En el caso de los servicios la balanza es un poco más favorable a los proveedores regionales, pero únicamente en servicios de gestión empresarial y capacitación y no en aquellos directamente vinculados con la producción, lo cual es una debilidad para lograr la integración de las cadenas de producción locales. 
La identificación de los sectores de actividad evidencia una economía poco diversificada, como lo corroboran el resto de los datos sobre el funcionamiento de la economía local. Además se caracteriza por una fuerte relación con los mercados externos, como cabía esperar, y podríamos hablar de dos áreas de la economía aisladas: la orientada al mercado externo — maquiladora—, y la que cubre el mercado interno, con diferencias importantes en su organización y funcionamiento. Este es uno de los efectos polarizadores que ha tenido el proceso de apertura económica en Baja California, donde se han reforzado los patrones tradicionales en lugar de originarse una reestructuración con otros factores de competitividad.

Retomando las alternativas utilizadas en la literatura sobre clústeres respecto a sus características trabajamos con acepciones geográficas del clúster y una visión horizontal del mismo. Estas estructuras responden a criterios de similitud, y desde nuestro punto de vista son agrupamientos en los que aún no están necesariamente muy desarrolladas las redes entre actores. Esta es de hecho la gran tarea pendiente en el caso de Baja California, donde las relaciones de complementariedad entre actores todavía no están desarrolladas y existen más bien acciones comunes en función de objetivos o necesidades compartidos.

La consolidación de estos clústeres incipientes, mediante la consolidación de las redes de actores, presenta un potencial de evolución hacia clústeres laterales — sectores entre los que existen posibles sinergias- para algunos de los grupos, y verticales — articulados en torno a cadenas de producción- en los menos. Esta situación es consistente con la juventud del estado, que explica que en muchos aspectos es todavía un sistema territorial joven en el que la infraestructura física y la organizacional son débiles. 
Los factores de competitividad que identifican los empresarios (Cuadro 4) son el coste y disponibilidad de mano de obra y la calidad de los productos fabricados, lo cual indica que no se han incorporado todavía las visiones de ventajas competitivas basadas en intangibles, ${ }^{12}$ sino más bien factores tradicionales de competitividad. Esta interpretación es reforzada por la respuesta obtenida respecto a las fortalezas que identifican tener los empresarios.

Cuadro 4. Factores de competitividad empresarial mencionados

\begin{tabular}{|l|c|c|c|c|}
\hline \multicolumn{1}{|c|}{ Factor } & Autopartes & Eléctrico & Plásticos & Textil \\
\hline Precio & $50.0 \%$ & $51.6 \%$ & $80.0 \%$ & $50.0 \%$ \\
\hline Tiempo de entrega & $50.0 \%$ & $48.4 \%$ & $53.3 \%$ & $33.3 \%$ \\
\hline Calidad de los componentes & - & $58.1 \%$ & $46.7 \%$ & $58.3 \%$ \\
\hline Tecnología & - & - & $26.7 \%$ & - \\
\hline
\end{tabular}

Fuente: Encuesta del proyecto Identificación y diagnóstico de las posibilidades de SPL en Baja California, financiado por SIMAC, Martínez 2006.

La identificación de debilidades abre algo más el panorama hacia posibles búsquedas de articulación, ya que entre ellas se mencionan la falta de asociaciones y colaboración entre empresarios, las estrategias de mercado inadecuadas, el crédito y la falta de información sobre tecnología, mercados y productos, todos ellos posibles campos de colaboración.

Las respuestas sobre los factores de competitividad empresarial de nuevo tienen la misma orientación, y la conclusión que obtenemos es que no se ha modificado la visión del empresariado local respecto a la organización de la producción en torno a la disponibilidad del factor trabajo, con los problemas que ello implica, en el sentido de que incide incluso en una intensificación del patrón de inmigración interna, con los subsecuentes problemas que esto genera. 
Partiendo de los modelos dominantes de clúster la orientación que parece factible para lograr la consolidación de los agrupamientos en Baja California es un híbrido del modelo de redes de PYMES, que podría tratar de integrar a algunas medianas y grandes empresas para incentivar la innovación, y del modelo de desarrollo regional que estimule una especialización a partir de redes de empresas o agrupamientos existentes o en formación. Esta hibridación permitiría coordinar las propuestas de acción y hacerlas congruentes entre sí, enlazando los objetivos de competitividad y de fomento de las PYMES con la incidencia sobre el desempeño económico y el desarrollo de la región; ambos modelos se refieren a los niveles micro y meso, aunque puede considerarse que los priorizan de forma diferente.

Cuadro 5. Los modelos internacionales dominantes de clúster

\begin{tabular}{|c|c|c|c|c|}
\hline & Nivel & $\begin{array}{c}\text { Propuesta } \\
\text { para la mejora }\end{array}$ & Actividad típica & Países típicos \\
\hline $\begin{array}{l}\text { Modelo nacional de } \\
\text { aprovechamiento }\end{array}$ & Mega/Meso & $\begin{array}{l}\text { Ventaja nacional } \\
\text { en ciertos sectores } \\
\text { o cadenas de valor }\end{array}$ & $\begin{array}{l}\text { Identificación de clústeres y } \\
\text { creación de condiciones de } \\
\text { apoyo }\end{array}$ & $\begin{array}{l}\text { Canadá, } \\
\text { Holanda } \\
\text { Dinamarca, } \\
\text { Finlandia, } \\
\text { Suecia }\end{array}$ \\
\hline $\begin{array}{l}\text { Modelos de redes } \\
\text { PYMES }\end{array}$ & Micro/Meso & $\begin{array}{l}\text { Competitividad- } \\
\text { PYMES }\end{array}$ & $\begin{array}{l}\text { Aumento en las } \\
\text { interacciones con los } \\
\text { portadores externos de } \\
\text { conocimiento para innovar y } \\
\text { aprender de otros }\end{array}$ & $\begin{array}{l}\text { Australia, } \\
\text { Nueva Zelanda, } \\
\text { Noruega, EEUU }\end{array}$ \\
\hline $\begin{array}{c}\text { Modelo de desarrollo } \\
\text { regional }\end{array}$ & Meso/Micro & $\begin{array}{l}\text { Actividad sobre el } \\
\text { desempeño } \\
\text { económico y el } \\
\text { desarrollo regional }\end{array}$ & $\begin{array}{l}\text { Estimulación de } \\
\text { especialización por medio de } \\
\text { inversión y de redes }\end{array}$ & $\begin{array}{l}\text { Canadá, EEUU, } \\
\text { Escocia (RU) } \\
\text { Gales (RU) }\end{array}$ \\
\hline $\begin{array}{l}\text { Modelos de enlace } \\
\text { sobre investigación } \\
\text { industrial }\end{array}$ & Micro/Meso & $\begin{array}{l}\text { Colaboración y } \\
\text { redes entre la } \\
\text { industria y la } \\
\text { investigación }\end{array}$ & $\begin{array}{l}\text { Creación de una masa crítica } \\
\text { en tecnologías emergentes, } \\
\text { atrayendo centros de } \\
\text { investigación, inversiones y } \\
\text { empresas }\end{array}$ & $\begin{array}{l}\text { Austria, } \\
\text { Alemania, } \\
\text { Países Bajos }\end{array}$ \\
\hline
\end{tabular}

Fuente: Boekholt 1999. 


\section{LA POLÍTICA DE FOMENTO Y DESARROLLO DE CLÚSTERES EN EL ESTADO DE BAJA CALIFORNIA}

El papel del gobierno local es preponderante en la consolidación de la estrategia de asociación o construcción de redes, en gran parte porque los actores perciben una situación de inseguridad jurídica que inhibe los proyectos de medio y largo plazo que serían donde hay mayor potencial de colaboración entre empresas y de las empresas con otras instancias de apoyo. A partir de 2002 el gobierno del estado, a través de la Secretaría de Desarrollo Económico, SEDECO, inicia los trabajos para articular una política de desarrollo estatal que involucre activamente a los empresarios; hasta ese momento la relación con las instancias gubernamentales se había circunscrito a la distribución de incentivos, mayoritariamente financieros, de los gobiernos estatal y federal; no existía una relación horizontal o articulación institucionalizada entre los actores del estado para impulsar el proyecto de desarrollo regional.

La nueva política de fomento de clústeres pretendió lograr la articulación horizontal incorporando a los empresarios al diseño, implementación y evaluación de la política de desarrollo empresarial, a pesar de los obstáculos existentes, y cambiar el esquema vertical de relaciones gobierno-actores económicos. La actividad de las instancias gubernamentales se planteó cómo fortalecer y desarrollar de manera organizada y cooperativa algunos de los sectores más importantes, estratégicos o emblemáticos, del estado. ${ }^{13}$ Uno de los aspectos importantes del planteamiento de esta estrategia fue incorporar en la planeación además de los sectores en que se especializa el estado a otros sectores productivos con bajo nivel de especialización y menor impacto en la economía regional en ese momento, pero con mayor identidad y arraigo locales como opciones para detonar el proceso de articulación de SPL de Baja California. 
Como primer paso se identificaron las vocaciones productivas ${ }^{14}$ del estado para a partir de ellas proceder a la identificación y diagnóstico cuantitativo de clústeres existentes y potenciales, y de sus fortalezas y debilidades. La parte cuantitativa de la identificación, mediante la matriz insumo/producto estatal, permitió evaluar la magnitud de las relaciones entre actividades e incluso en algunos casos detectar vínculos que no se habían contemplado. La eficiencia de la política, una vez hecha la identificación de los clústeres reales y potenciales, se fundamentó en la selección de instrumentos ad hoc a la situación revelada por cada diagnóstico y la priorización de las acciones sobre los puntos estratégicos de los clústeres. La evaluación de la eficacia se haría partiendo del grado de comunicación y cooperación desarrollados, por esta razón los acuerdos de cooperación han sido cruciales desde el inicio hasta la fecha. La evaluación de impacto requiere algo más de tiempo para registrar cambios en la base productiva bajacaliforniana.

Se puede considerar que los tres principales cambios en el enfoque de esta nueva política fueron: 1) que las unidades o sujetos de políticas pasaron a ser redes de empresas, clústeres, en lugar de empresas individuales, 2) que se propuso enfatizar la provisión de servicios reales a las empresas en lugar de incentivos económicos, y 3) que se partió de un diagnóstico que conjugó los aspectos cualitativos de la detección de vocaciones y los cuantitativos de la cuantificación de encadenamientos. Los apoyos inicialmente se condicionaron a que el clúster estuviese identificado y contase con un plan estratégico ${ }^{15}$ que contemplara la línea de acción para la que se iba a solicitar el apoyo, y que de la acción se beneficiasen al menos diez empresas. Estas características contribuyeron a que la política de desarrollo empresarial basada en el desarrollo y fomento de clústeres de Baja California se considerase de las pioneras en su ámbito en el país. 
Inicialmente, se identificaron los clústeres de turismo, electrónica, software, automotriz y plásticos, vitivinicultura, muebles, horticultura y cerveza, es decir, ocho, y aunque aún es pronto para evaluar en todas sus dimensiones la política de fomento y desarrollo de clústeres, un primer dato es que desde 2003 a enero de 2008 el número de clústeres registrados ha aumentado a quince: aeroespacial, agroindustrial, automotriz, biotecnología, electrónica — display devices_, energía, logística, mueble y madera, pesca y acuacultura, productos médicos, plásticos, servicios médicos, tecnologías de información, turismo y vitivinícola. Se mantuvieron activos los agrupamientos iniciales y surgieron otros cinco.

La comparación de las listas de clústeres de Baja California desde 2003 a 2008 refleja, en primera instancia, una reorganización de parte de los grupos que ya existían, de manera que los actores asociados en cada clúster se modificaron según el funcionamiento del sistema productivo en la realidad o según los intereses de parte de los integrantes de las actividades. Esto último se dio particularmente en casos en los que coexistieron actividades maduras con actividades más innovadoras, lo que permitió identificar las ventajas de la cooperación con vistas a generar sinergias y economías externas de diversos tipos. Son los casos en que la complementariedad de los involucrados en el clúster es el elemento crítico de la cooperación.

El segundo cambio que reflejan estas listas de clústeres es el tipo de agrupamientos que surgen, ya que se podría decir que la «segunda ola» está integrada por clústeres de actividades más intensivas en tecnología y conocimiento — biotecnología, aeroespacial— y clústeres de servicios a la actividad en general —energía y logística—. Una primera interpretación de esto podría contemplar que se está dando un cambio 
estructural en la base económica de la región, en la que están repuntando los sectores de potencial especialización o actividades incipientes y que está organizándose de manera más integrada el sistema productivo local, con lo que surgen demandas de servicios más avanzados a las empresas —energéticos y logística—. Para corroborar esta interpretación sería necesario contar con la información precisa sobre los proyectos de cada clúster y las relaciones entre agrupamientos, así como seguir la evolución tanto de los agrupamientos, ya actores del sistema económico regional, como de las relaciones entre ellos en una dinámica de integración de la economía regional como sistema productivo local. ${ }^{16}$

Entre las líneas de acción o iniciativas planteadas formalmente por los clústeres de Baja California en sus planes estratégicos destacan, en primer lugar, los proyectos en el ámbito de la formación de recursos humanos. Esta es una de las líneas de acción presente en prácticamente todos planes, y abarca desde la capacitación de los operadores y técnicos de las empresas hasta la capacitación en temas de administración empresarial para los gestores y empresarios. Tal demanda de las empresas es congruente con dos características de la región: en primer lugar, la presencia de un gran número de actividades que fundamentan su competitividad en los costos y disponibilidad de la mano de obra; y en segundo lugar, el escaso impacto de la industria que se ha desarrollado hasta el momento en la orientación del mercado laboral hacia competitividad por calidad.

Otra línea de acción que retoman la mayor parte de los planes estratégicos es la de desarrollo de proveedores o articulación con clientes, lo cual indica que al menos parte de las empresas de la región ya busca trabajar en el camino de la integración de redes, más o menos locales según los casos, con una visión de coordinación y cooperación algo más compleja que el simple intercambio de productos, mercancías o servicios. En algunos de 
los planes incluso se plantea una línea estratégica de vinculación entre los actores del mismo sector.

En la lógica de esta cooperación-colaboración de diferentes maneras surge otra preocupación, que se podría considerar general por aparecer en la mayoría de los planteamientos que hacen los agrupamientos empresariales bajacalifornianos: la necesidad de articularse con otras instituciones que denominan de apoyo, entre las que se encuentran de manera destacada el sector gobierno y el educativo de investigación. El gobierno aparece considerado como un actor importante en la articulación de los grupos — correspondiendo con el papel de motivador de los clústeres que tuvo con el inicio de la política一, como posible financiador de parte de las acciones y sobre todo como un interlocutor para mejorar el marco regulatorio e institucional en el que se desenvuelven las actividades.

Todo lo anterior, sin ser una evaluación en sentido estricto de la política de desarrollo empresarial basada en el desarrollo y fomento de clústeres en Baja California, permite sugerir que si aún no se puede hablar de un sistema productivo local bajacaliforniano, sí comienzan a darse sinergias en algunas actividades y entre actividades, como lo refleja la permanencia de los agrupamientos iniciales y el surgimiento de nuevos, y empieza a permear en un sector de la base económica la visión de un esquema de cooperación para lograr mayor competitividad en los ámbitos sectorial y territorial. 


\section{REFLEXIONES FINALES}

En el caso de Baja California el origen, local o internacional, de las empresas agrupadas y la forma de organización inicial de las mismas han sido determinantes de las posibilidades de existencia y refuerzo de articulación entre los actores. Por esta razón la estrategia de vinculación de la economía estatal aparentemente ha tenido mayor impacto y presenta mayores posibilidades de éxito en los sectores de actividad emergente con alto potencial de crecimiento que pueden consolidarse con una lógica de competitividad basada en la cooperación y la calidad.

El análisis de las redes — por agrupamiento— resalta el rezago en la adaptación institucional a las dinámicas de abajo arriba como uno de los principales inhibidores a los que tienen que hacer frente los actores locales. La obsolescencia institucional se manifiesta por igual en el ámbito público y en el privado, y apunta la inexistencia de un consenso sobre la organización más adecuada para lograr los objetivos de desarrollo económico planteados para el estado a partir de un consenso público-privado. Esta situación responde a lo reciente que han sido las dinámicas de descentralización y apertura en el país, lo que explica que el aprendizaje de las nuevas reglas es un proceso todavía incipiente, aunque ya presente sobre todo en los estados que como Baja California enfrentan la competitividad internacional de forma más directa por su ubicación y por su dinámica de producción manufacturera muy marcada por la presencia de las maquiladoras.

En el caso de México, y en particular de Baja California, los resultados que se han obtenido de diferentes investigaciones ${ }^{17}$ demuestran: 1) que el proceso de integración ha reforzado los modelos de especialización existentes, y 2) que en Baja California el grado 
de articulación entre actores para poder hablar de sistemas productivos locales es aún bajo, no obstante presenta elementos con los que consolidar un sistema productivo local que responda a las especificidades regionales.

Por lo tanto se ha dado una situación de crecimiento sostenido, sin que hayan ido a la misma velocidad los cambios estructurales de la base productiva regional, que permitiría hablar de un desarrollo del potencial competitivo regional. En este sentido es necesario un replanteamiento de la estrategia de desarrollo del estado para iniciar y establecer una dinámica que apunte a resultados de largo plazo —desarrollo— y no tanto de beneficios cortoplacistas — crecimiento - fundamentada en los recursos regionales y no solo en la inversión externa. Es difícil hablar de que la empresarialidad local ha logrado consolidar una senda de desarrollo local o un modelo de desarrollo que contribuya a la construcción territorial del estado.

Por lo tanto, en el caso de Baja California, a pesar de la existencia de los intercambios detectados a partir de la tabla o matriz de insumo/producto y de la especialización del estado en ciertos sectores, todavía no se han generado las redes de interacción que permitan explotar realmente las economías externas. La mención por parte de una proporción de empresarios de la necesidad de mayor cooperación interempresarial y con otras instituciones es un indicio de que ya existe cierta conciencia de la necesidad de articulación para lograr mayores niveles de competitividad.

La política de desarrollo empresarial basada en el desarrollo y fomento de clústeres podría ser un buen inicio de este cambio de enfoque hacia una propuesta de desarrollo local marcada por la evolución dirigida a la consolidación de un sistema productivo local con características propias. Los fundamentos de los que parte engloban 
la mayoría de los elementos necesarios para lograr el objetivo de integración del sistema productivo local, falta ver si los actores involucrados deciden darle un voto de confianza y el tiempo y recursos necesarios para lograr los objetivos comunes y perpetuar el establecimiento de nuevos acuerdos de cooperación. 


\section{BIBLIOGRAFÍA}

Aghon, Gabriel, Francisco Alburquerque y Patricia Cortés, 2001, Desarrollo económico local y descentralización en América Latina: Análisis comparativo. Proyecto Regional de Desarrollo Económico Local y Descentralización. CEPAL, GTZ, Chile.

Becattini, Giacomo, 1979, «Dal settore industriale al distretto industriale: alcune considerazione sull l'unita d'indagine dell'economia industriale». Revista di Economia e Politica Industriale, núm. 1, pp. 7-21.

2005, La oruga y la mariposa. Un caso ejemplar de desarrollo en la Italia de los distritos industriales: Prato (1954-1993). Universidad de Valladolid, Valladolid.

Boekholt, Thuriaux Pierre, 1999, Overview of Cluster policies in international perspective. A report for the Dutch ministry of Economic Affair. Technopolis, The Hague.

Camagni, Roberto, 1992, «Development Scenarios and Policy Guidelines for the Lagging Regions in the 1990s». Regional Studies, vol. 26, núm. 4, pp. 361-374.

Castañeda, Vicente, 1991, «México: cuarenta años de acción estatal sobre el territorio». Revista Interamericana de Planificación, vol. XXIV, núm. 94, pp. 208-225.

Colletis, Gabriel y Bernard Pecqueur, 1993, «Les facteurs de la concurrence spatiale et de la construction des territoires». En Notes de travail 11, IREPD, mars. Université Mendés France, Grenoble. 
Garofoli, Gioacchino (editor), 1992, Endogenous Development and Southern Europe. Avebury, Aldershot.

1993, «Economic Development, Organization of Production and Territory». Revue d'économie industrielle, núm. 64, pp. 22-37.

Martínez, Sárah, 2006, «Sistemas productivos locales e integración económica: el caso de Baja California, México». Tesis doctoral. Universidad Autónoma de Madrid, Madrid.

Martínez, Sárah y Noé Arón, 2001, «Identificación y diagnóstico de las posibilidades de SPL en Baja California». Reporte de investigación, financiado por SIMAC.

Massey, Doreen, 1983, «Industrial Restructuring as Class Restructuring: Production Decentralization and Local Uniqueness». Regional Studies, vol. 17, núm. 2, pp. 73-89.

Nelson, Robert y Bernard Sampat, 2001, «Making sense of institutions as a factor shaping economic performance». Journal of Economic Behaviour and Organization, núm. 44, pp. 31-54.

Pacheco-Vega, Raúl, 2007, «Una crítica al paradigma de desarrollo regional mediante clústers industriales forzados». Estudios Sociológicos, vol. XXV, núm. 75, pp. 683-707.

Perrin, Jean Claude, 1974, Le développement régional. Presses universitaires de France, Paris.

Precedo, Andrés, 2004, Nuevas realidades territoriales para el siglo XXI. Desarrollo local, identidad territorial y ciudad difusa. Editorial Síntesis, Madrid. 
Vázquez, Antonio, 1992, «Local Development Initiatives Under Incipient Regional Autonomy: The Spanish Experience During the Eighties». En Endogenous Development and Southern Europe, pp. 165-183, editado por Gioacchino Garofoli. Avebury, Aldershot.

2002, «Crecimiento endógeno o desarrollo endógeno?». En Desarrollo local: teorías y estrategias, pp. 83-106, coordinado por Giacomo Beccatini, María, Teresa Costa y Joan Trullén. Diputació de Barcelona,Civitas, Barcelona.

2005, Las nuevas fuerzas del desarrollo. Antoni Bosch editor, Barcelona. 


\section{Notas}

${ }^{1}$ Este artículo retoma parte de los resultados del trabajo de investigación doctoral de la autora titulado «Sistemas productivos locales e integración económica: el caso de Baja California México». Agradezco los enriquecedores comentarios del Dr. Antonio Vázquez Barquero, director del trabajo de tesis, y de los doctores Enrique Cabrero y Noé Arón Fuentes.

${ }^{2}$ El estado ocupa la mitad norte de la península de California, en el extremo noroeste del país. Su localización contigua a Estados Unidos y en particular a una de las economías más dinámicas del mundo, la californiana, ha sido su principal ventaja respecto al resto de la República.

${ }^{3}$ Se utiliza aquí la denominación «regiones funcionales» para hacer referencia a que son sistemas integrados en aspectos medulares de su funcionamiento. Según el grado de integración de hecho, esos aspectos de funcionamiento interrelacionados serán más o menos en número y se escalonarán, cualitativamente, desde aspectos meramente instrumentales a procesos de decisión.

${ }^{4}$ Estos elementos son la base del cuestionario que se aplicó a las empresas de Baja California para llevar a cabo el estudio de caso.

${ }^{5}$ El término reestructuración productiva está referido a la reestructuración industrial que D. Massey define como «uno de los mecanismos a través de los que se reforma la estructura social y se cambian las relaciones sociales, y se rompen o reconstruyen las bases de la acción política» (1983: 74).

${ }^{6}$ Pacheco-Vega (2007) enfatiza como tales el canibalismo empresarial, la excesiva especialización y la saturación de mercados, que pueden derivarse de la existencia de sistemas productivos dependientes de un clúster monoespecializado.

${ }^{7}$ Sárah Martínez Pellégrini, 2006, «Sistemas productivos locales e integración económica: el caso de Baja California, México». Tesis doctoral, cap. 4. Universidad Autónoma de Madrid, España.

${ }^{8}$ No se abunda más en este punto porque es tema ya recurrente en las discusiones sobre desarrollo. Es suficiente revisar los índices de desarrollo humano y los índices de Gini que suelen acompañarlos para confirmar este particular. Se plantea de manera extensa en Martínez 2006, cap. 4.

${ }^{9}$ Estamos aplicando la clasificación regional propuesta en Vázquez 1992.

${ }^{10}$ Los datos que se presentan son resultado de la aplicación de entrevistas y encuestas a 150 empresas bajacalifornianas seleccionadas como muestra estratificada para identificar su organización e interrelaciones. La metodología puede consultarse en Martínez 2006.

${ }^{11}$ Los aspectos que se analizan de la organización de los agrupamientos se documentaron a partir de fuentes secundarias y de la aplicación de cuestionarios y entrevistas en 150 empresas bajacalifornianas de los sectores autopartes, textil, de plásticos y eléctrico-electrónico.

${ }^{12}$ Estos intangibles se refieren fundamentalmente al capital humano y a la capacidad de organización del sistema en sus diferentes niveles institucionales, incluyendo los mecanismos de cooperación formal e informal entre actores. En general podríamos decir que son todos los factores, no acotados a los cambios tecnológicos, susceptibles de incorporar innovaciones en el funcionamiento del sistema regional.

${ }^{13}$ Las actividades importantes son aquellas que presentaban empleo y PIB por encima del promedio estatal; las estratégicas aquéllas cuyo crecimiento había sido superior al promedio estatal, y las emblemáticas las que se consideraban distintivas del estado, como por ejemplo la vitivinicultura. El clúster vitivinícola ha sido considerado uno de los emblemáticos de Baja California, produce $80 \%$ del vino del país presentando estándares de calidad que lo han hecho acreedor de numerosos premios internacionales y a posicionarse en el mercado internacional a pesar de su reducida producción. Ver «Plan estratégico del clúster vitivinícola» (2003), publicación de la Secretaría de Desarrollo Económico del Estado de Baja California, responsable del proyecto Sárah Martínez Pellégrini.

${ }^{14}$ Realizado por Integra Internacional cuyos resultados se encuentran en «La política de desarrollo empresarial del estado: vocaciones productivas», para SEDECO.

${ }^{15}$ Los planes estratégicos de los agrupamientos pueden consultarse en www.clusterbc.org, así como otra información sobre sus actividades e integración.

${ }^{16}$ Hasta la fecha solo existe información sistemática sobre el monto de inversión por clúster, según los proyectos financiados total o parcialmente con participación del sector público.

${ }^{17}$ Ver Martínez 2006. 
Revista Pueblos y Fronteras digital

Iniciativa empresarial y desarrollo local en América Latina
Núm. 6, Diciembre 2008 - Mayo 2009

http:// www.pueblosyfronteras.unam.mx

Fecha de recepción: 31 de enero de 2008

Fecha de aceptación: 25 de abril de 2008 